\title{
A Novel Shim-Assisted Resistance Spot Welding Process to Improve Weldability of Medium-Mn Transformation-Induced Plasticity Steel
}

\begin{abstract}
SHUOSHUO LI, SHANGLU YANG, QI LU, HAIWEN LUO, and WU TAO
Medium-Mn transformation-induced plasticity steels have great potential to significantly reduce vehicle weight and improve fuel economy due to their outstanding combination of high strength and excellent ductility. One bottleneck to the application is their poor weldability resulting from their high Mn contents. In this paper, three resistance spot welding set-ups, including no shim, an interstitial-free steel shim at the faying interface (shim-in) and shims against the electrodes (shim-out), were incorporated to investigate the weldability of Fe-7Mn-0.14C medium-Mn steel. Tensile-shear, cross-tension, and microhardness tests were used to evaluate the mechanical properties of the welds. Experimental results demonstrated that the failure mode of the welds transitioned from the interfacial fracture in the case of no shim to the desired nugget pull-out fracture in the shim-out set-up, resulting in dramatical improvements in both peak loads and their corresponding extensions during the tensile-shear and cross-tension tests. In contrast, the shim-in set-up made no improvement. What can contribute to such improvement was then discussed on the basis of observed morphologies and microstructures of welds.
\end{abstract}

https://doi.org/10.1007/s11663-018-1463-9

(C) The Author(s) 2018

\section{INTRODUCTION}

THE application of advanced high strength steels (AHSS) to automotive industries can significantly reduce vehicle weight and improve fuel economy. Compared to the $1^{\text {st }}$ generation and the $2^{\text {nd }}$ generation AHSS, the 3rd generation (Gen.3) AHSS have a better combination of high strength and superior ductility; therefore, they receive an increasing interest from both steel and automotive industries. ${ }^{[1]}$ Medium-Mn TRIP steels, which usually contain approximately 5 to $12 \mathrm{wt}$ pct $\mathrm{Mn}$, are considered one of the most promising candidates of the Gen.3 AHSS. They have a dualphased microstructure of ferrite and retained austenite; the latter is often in the range of 20 to 50 pct. ${ }^{[2]}$ Both

SHUOSHUO LI and HAIWEN LUO are with the School of Metallurgical and Ecological Engineering, University of Science and Technology Beijing, Beijing 100083, P.R. China. Contact e-mail: luohaiwen@ustb.edu.cn SHANGLU YANG and WU TAO are with the Shanghai Institute of Optics and Fine Mechanics, Chinese Academy of Sciences, Shanghai 201800, P.R. China. Contact e-mail: yang_unitech@126.com QI LU is with the China Science Lab, General Motors Global Research \& Development Center, Shanghai 200120, China.

Manuscript submitted September 26, 2018.

Article published online December 6, 2018. strength and ductility could be improved due to the so-called transformation-induced plasticity (TRIP) effect, resulting from the transformation of the retained austenite to martensite during the tensile deformation. ${ }^{[3-5]}$ It has been reported that the product of strength and ductility of many developed medium-Mn steels can be more than $30,000 \mathrm{MPa}$ pct. ${ }^{[6]}$

The weldability of medium-Mn TRIP steels has to be assessed before they can be passed to commercial applications. In particular, the mechanical properties of welds play essential roles in crash performance. Some efforts have been recently made to investigate the microstructure and mechanical properties of welded medium-Mn TRIP steels after using different welding processes. For examples, a $\mathrm{Fe}-8 \mathrm{Mn}-0.06 \mathrm{C}$ (all are in weight percentage, unless mentioned elsewhere) steel was joined by gas tungsten arc welding, and the impact toughness of full martensite structure in weld was evaluated, which strongly depends on the sizes of the martensitic block and packet. ${ }^{[7]}$ Gas metal arc welding was applied to $\mathrm{Fe}-6.5 \mathrm{Mn}-0.98 \mathrm{C}$ steel with the added pulse current, which can enhance the plastic deformation capacity of weld joint. ${ }^{[8]}$ Laser welding of Fe-10.4Mn-0.15C steel produced a fusion zone (FZ) of welds, which were mainly composed of martensite and some interdendritic austenite, and no softening in the heat-affected zone (HAZ) ${ }^{[9]}$ In addition, the precipitation of VC nanoparticles could lead to the increases of both microhardness and tensile strength of the simulated HAZ in the Fe-8.1Mn-0.98C steel. ${ }^{[10]}$ 
However, we are still facing a great challenge in designing a proper weld process to obtain the good-quality welds with the satisfied properties for the medium-Mn TRIP steels because the brittle and hard phase is frequently formed during welding due to their high Mn content, which leads to a high susceptibility to cracking. ${ }^{[11]}$ In the automotive industry, moreover, resistance spot welding (RSW) is a primary and vital sheet metal joining process due to the two advantages: the high welding speed and the excellent suitability for robotization. ${ }^{[12,13]}$ There are about 3000 to 5000 spot welds in a typical steel body-in-white. ${ }^{[14]}$ During the RSW process, the thermal process destroys the designed microstructure of AHSS that has been formed during the previous rolling and annealing processes, which may deteriorate the mechanical properties of welds. ${ }^{[12]}$ In particular, the interfacial fracture (IF) of weld after the RSW of AHSS is detrimental to the crashworthiness of the vehicles and shall be avoided. ${ }^{[15]}$ Peterson has used a low carbon dilution sheet during the RSW of martensitic steel sheets to change the IF fracture mode. ${ }^{[16]}$ However, there are few researches published about the RSW of medium-Mn steels, to our best knowledge.

In this paper, different RSW processes were used to weld a medium-Mn steel received from a commercial supplier after a trial industrial production. Three resistance spot welding set-ups, including no shim, an interstitial-free steel shim at the faying interface (shim-in) and shims against the electrodes (shim-out), were implemented to evaluate the weldability of medium-Mn steel. The effects of the interstitial-free steel shims at different locations on the mechanical performance were analyzed through the morphology and microstructure of weld, microhardness measurement, and tensile-shear tests.

\section{EXPERIMENTAL PROCEDURES}

The studied medium-Mn TRIP steel is a 1.4-mm-thick Fe-7.14Mn-0.14C steel (7Mn steel) sheet with no coating, whose exact chemical composition and mechanical properties are listed in Table I. In addition, the interstitial-free (IF) steel shim was employed in some welding set-ups for improved weldability, whose composition and mechanical properties are also given in Table I. The microstructure of received steel is the mixture of lamellar ferrite, ultrafine austenite, and some cementite, as shown in Figure 1. The conventional RSW set-up having no shim, the welding set-up with a $0.8-\mathrm{mm}$-thick uncoated IF steel shim at the faying interface (shim-in) and the one with two IF shims against the electrodes (shim-out) are shown in Figures 2(a), (b), and (c) respectively. They were all implemented to assess the weldability of the studied steel.

RSW experiments were performed using a mediumfrequency direct current (MFDC) resistance welding machine, WT6000. The welding schedule in Figure 3 was employed for all the three welding set-ups. 16-mm-diameter domed electrodes with a 7.0-mm-diameter flat tip end, made of $\mathrm{Cu}-0.2 \mathrm{Zr}$ (wt pct) alloy, were used. Both the tensile-shear and cross-tension tests were conducted to evaluate the mechanical behaviors of spot welds according to ANSI/AWS/SAE/D8.9-97 standards. The dimensions of the tensile-shear and cross-tension specimens are shown in Figure 4 according to the general motor (GM) manufacturing engineering standard. Both tensile-shear and cross-tension tests were carried out at the crosshead speed of $2 \mathrm{~mm} / \mathrm{min}$ using an Instron 5982 testing machine. A minimum of three replicates were tested for each condition. After failure, the fracture surfaces were examined by a scanning electron microscopy (SEM, ZEISS AURIGA). The welds were sectioned, ground, polished, and etched in 4 pct nital for microstructure examinations under optical microscope (OM, AEISS Axio Imager Z2 m), SEM with a mounted energy dispersive spectrometer (EDS). The average size of nugget was measured from at least three replications on the cross-sectional view of welds. The elemental enrichment and distribution were examined using electron probe microanalyzer (EPMA). Vickers microhardness was measured at the load of $500 \mathrm{~g}$ and a dwell time of 10 seconds.

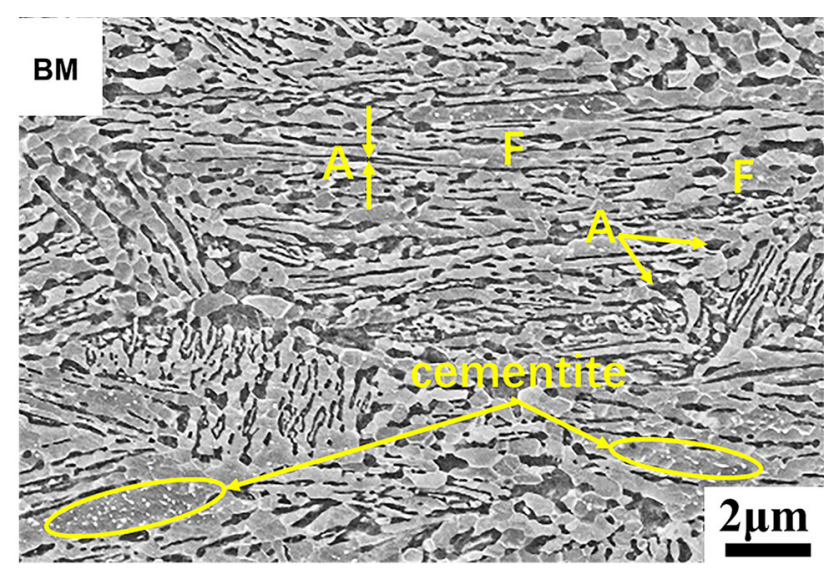

Fig. 1-Microstructures of the received 7Mn steel (BM, base metal).

Table I. Chemical Compositions and Mechanical Properties of the Studied 7Mn Steel and IF Steel Shim

\begin{tabular}{|c|c|c|c|c|c|c|c|c|c|}
\hline \multirow[b]{2}{*}{ Steel Grade } & \multicolumn{6}{|c|}{ Composition (Wt Pct) } & \multicolumn{3}{|c|}{ Mechanical Properties } \\
\hline & $\mathrm{Mn}$ & $\mathrm{C}$ & $\mathrm{Si}$ & $\mathrm{P}$ & $\mathrm{S}$ & $\mathrm{Fe}$ & UTS (MPa) & YS (MPa) & TE (Pct) \\
\hline $7 \mathrm{Mn}$ & 7.14 & 0.14 & 0.23 & 0.056 & - & bal. & 1100 & 1080 & $35-40$ \\
\hline IF & 0.099 & 0.001 & 0.004 & 0.079 & 0.0067 & bal. & 294 & 140 & 44.1 \\
\hline
\end{tabular}




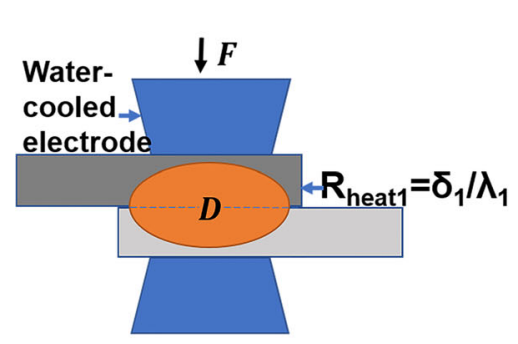

(a)

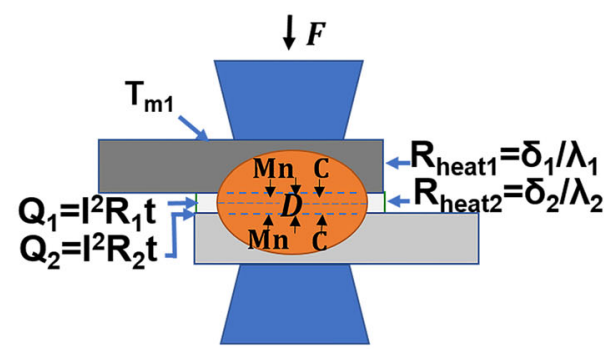

(b)

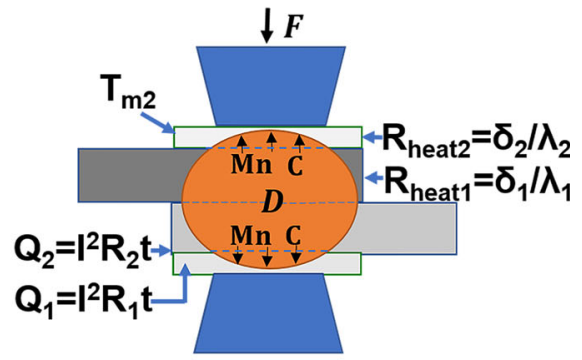

(c)

Fig. 2-Illustrations of three welding set-ups employed in the study. (a) No shim, $(b)$ shim-in, i.e., a 0.8-mm-thick uncoated IF steel shim at the faying interface, $(c)$ shim-out, i.e., two IF shims against the electrodes $\left(F\right.$, electrode force; $D$, nugget; $T_{\mathrm{m}}$, solidus temperature; $Q$, heat quantity; $R$, electrical resistance; $R_{\text {heat }}$, heat resistance; $\delta$, material thickness; $\lambda$, thermal conductivity. All of them will be discussed later).

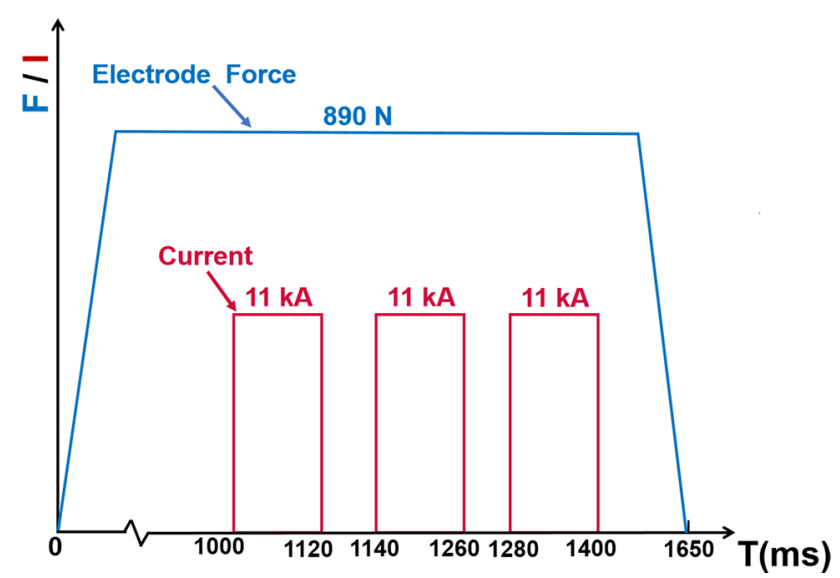

Fig. 3-The resistance spot welding schedule used in the three welding set-ups.

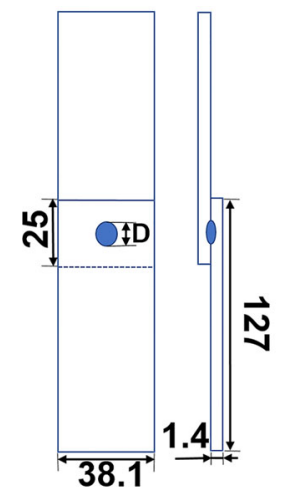

(a)

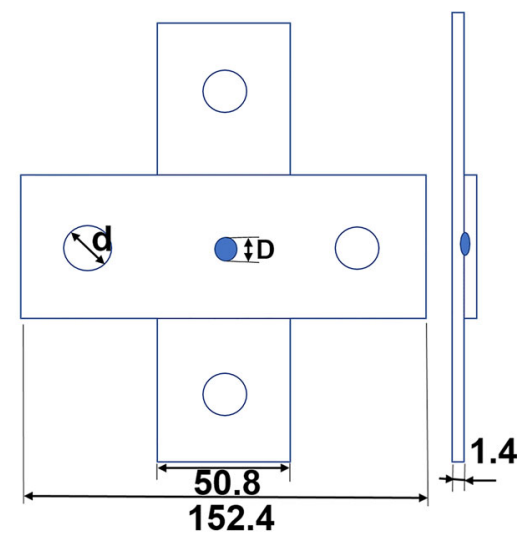

(b)
Fig. 4-The dimensions of specimens $(\mathrm{mm})$ for the tensile-shear test (a) and the cross-tension test $(b), d=18 \mathrm{~mm}$.

\section{RESULTS}

\section{A. Morphology and Microstructure of Welds}

The weld surface quality and shapes of nuggets obtained in the three welding set-ups are shown in Figure 5. Both splash and cracking were observed in the no-shim set-up (Figure 5(a)). In contrast, the shim-in set-up produced the largest expulsion on the surface, the most porosities and the severest cracking (Figure 5(b)), whilst the shim-out one led to the largest nugget and the best surface quality. Figure 6 shows that both the measured diameter and thickness of nugget in the shim-out set-up are largest, and the diameter of shim-in nugget is smallest, while the thickness of no-shim nugget is smallest.

Figure 7 shows the microstructures and the distribution of Mn in both HAZ and FZ. It can be seen that the Mn content of FZ in the case of shim-out set-up was slightly lower than that of HAZ but it is lowest in the shim-in set-up, the latter is clearly attributed to the entire IF steel shim contributing to the dilution of $\mathrm{Mn}$ content. It could be deduced that the $\mathrm{C}$ content in FZ of the shim-out and shim-in set-up should be also reduced due to the ultra-low $\mathrm{C}$ content of the IF steel shim, which may cause the microstructural change in the fusion zone. Figure 8 shows the microstructures of FZ in the three set-ups. They are composed of mainly martensite and a very small fraction of austenite. Therefore, it appears that the microstructures of FZs actually do not change with different $\mathrm{C}$ and $\mathrm{Mn}$ contents in the three set-ups.

\section{B. Mechanical Properties}

Figure 9 shows that the Vickers microhardness profiles across the welds have the similar distribution in the three set-ups. The microhardness in HAZs is similar in all the three set-ups, and it is much higher than that in 

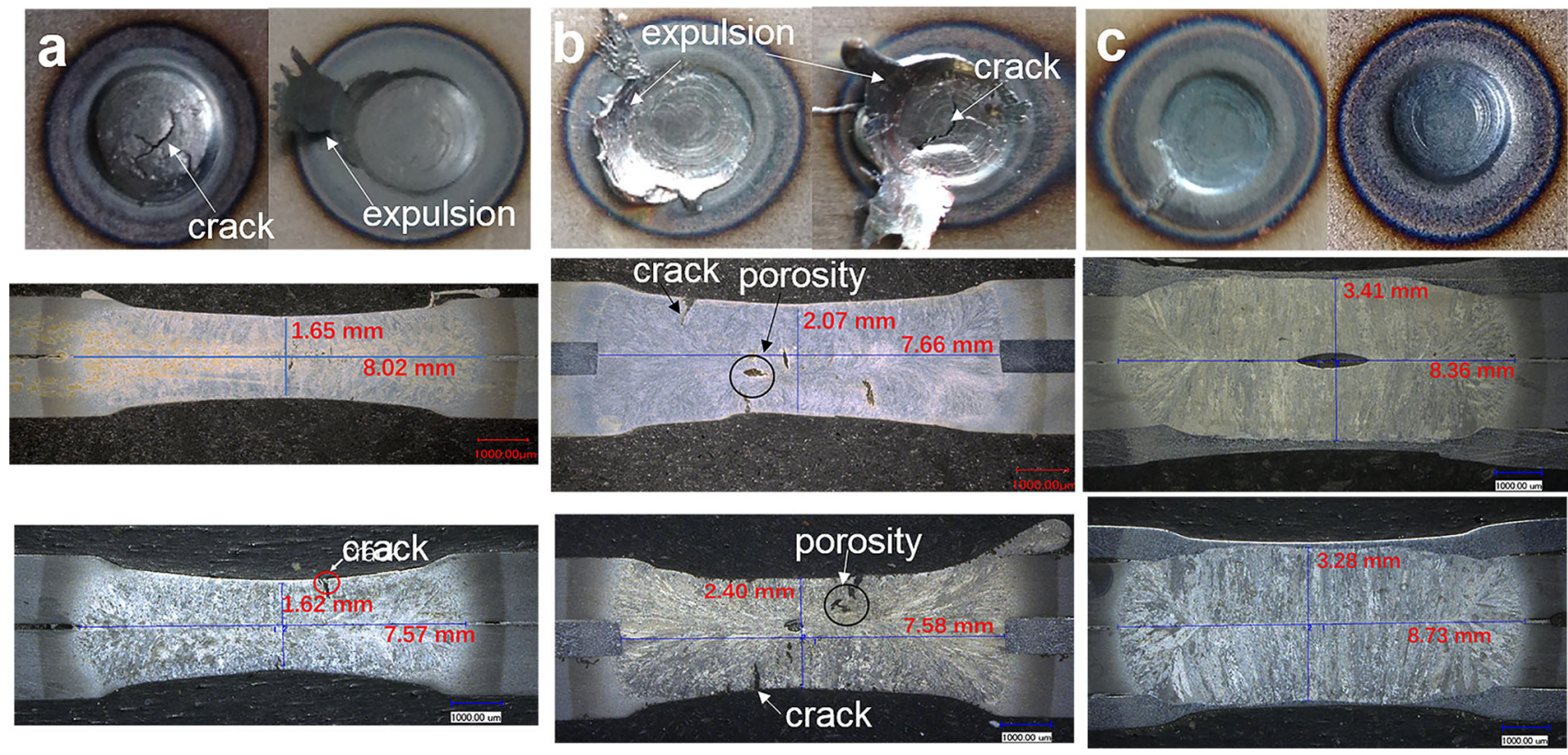

Fig. 5-The nuggets and weld surface obtained in the three welding set-ups. (a) No shim, $(b)$ shim-in, $(c)$ shim-out.

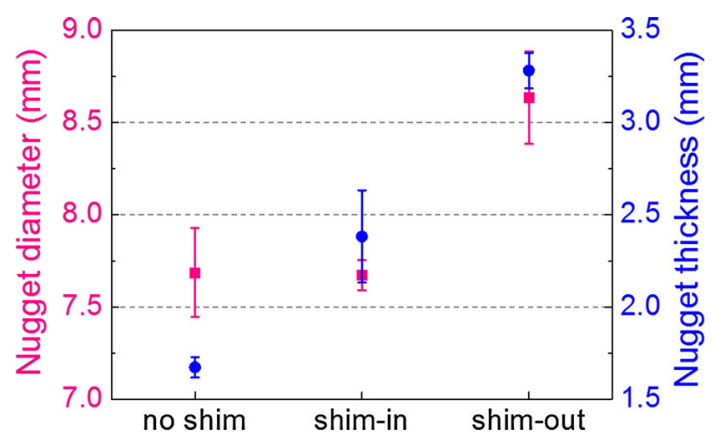

Fig. 6-The comparison of nugget diameters and thickness obtained in the three welding set-ups.
FZs; moreover, the microhardness in the FZ of shim-in set-up is lowest. This is consistent with the microstructural observations in Figures 7 and 8 , because the hardness of HAZ is actually determined by the martensite formed in the medium $\mathrm{Mn}$ steel so that they are similar in the three set-ups; whilst the hardness of FZ is determined by the martensite formed with different compositions, the latter depends on the mixing extent of IF and medium Mn steel melt during welding. Obviously, the shim-in setup produced the complete melting of the IF steel shim whose melt was then merging to the $7 \mathrm{Mn}$ steel melt, leading to the lowest contents of both $\mathrm{C}$ and $\mathrm{Mn}$ due to the strongest dilution; subsequently, the lowest microhardness in the FZ was achieved in this welding set-up, as observed in Figure 9.
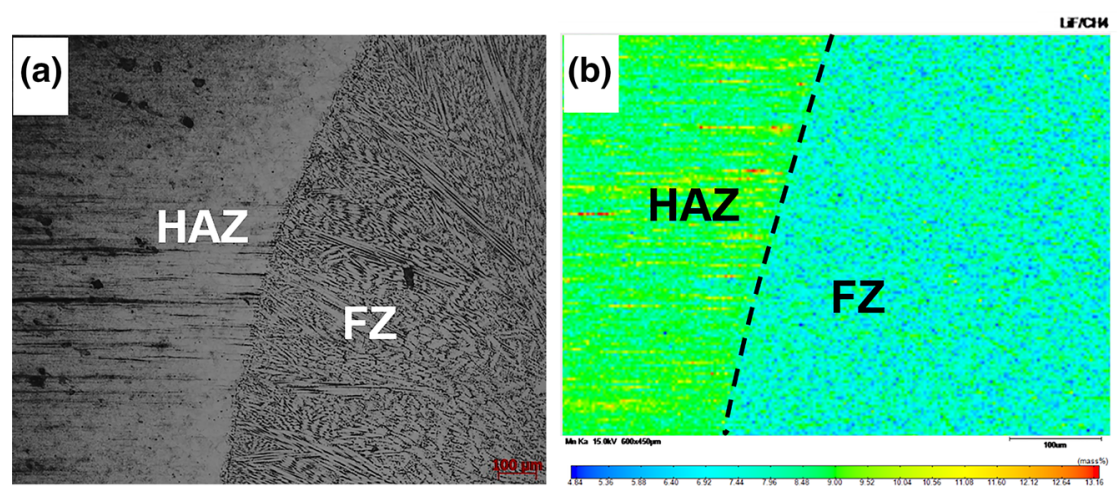

(c)

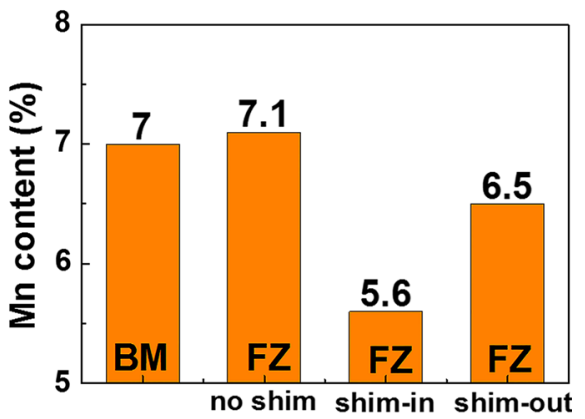

Fig. 7-Microstructural and compositional examination across the welds. (a) Microstructures in the shim-out welding set-up, (b) mapping of Mn distribution by EPMA in the shim-out set-up, $(c)$ the average Mn contents in BM, FZ of three set-ups measured by EDS. 

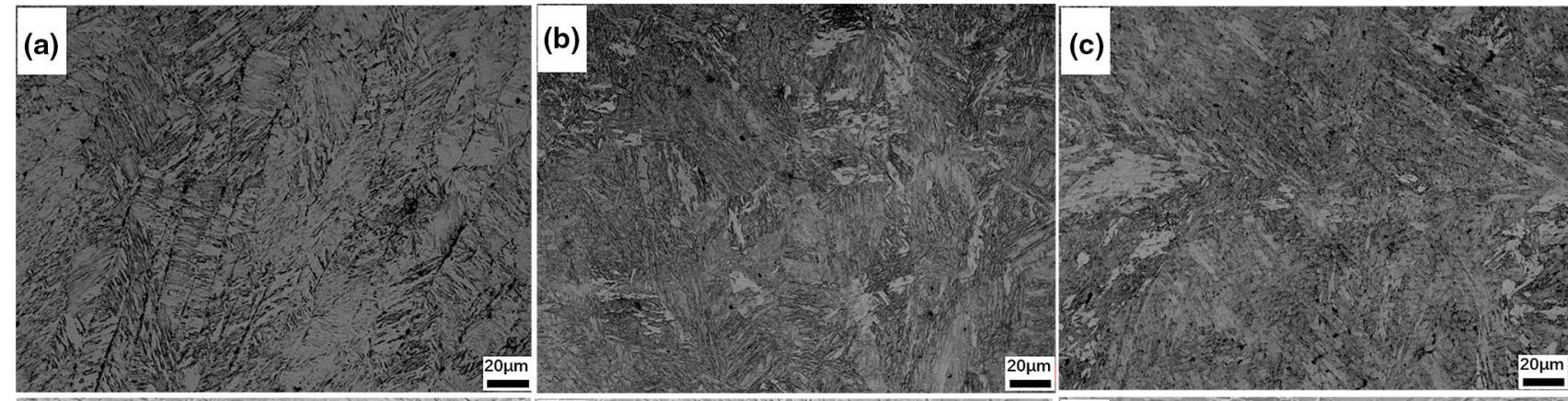

(d)

(e)

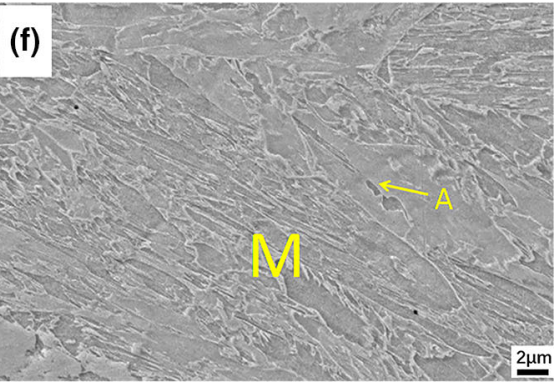

Fig. 8-The microstructures of fusion zones examined by optical microscopes ( $a$ to $c$ ) and SEM ( $d$ to $f$ ). (a and d) No shim, (b and e) shim-in, (c and f) shim-out.

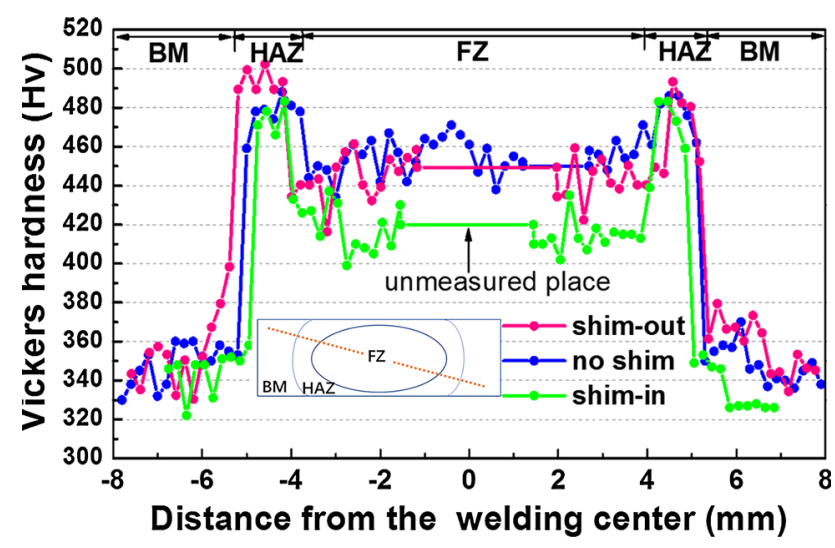

Fig. 9-The microhardness distributions in the welded zones along diagonal direction.

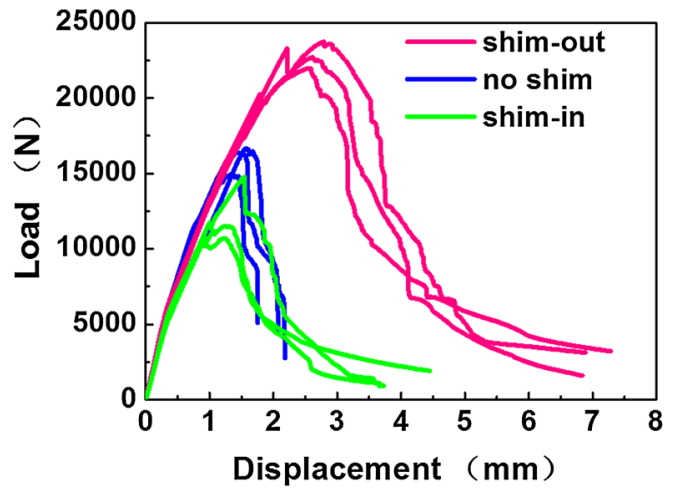

(a)
Figure 10 shows the load-displacement curves during the tensile-shear (TS) and cross-tension (CT) tests for the three welding set-ups. Clearly, the conventional no shim set-up produced the lowest values of both peak loads and extensions in both the TS and CT tests, whilst the shim-out set-up led to the best mechanical properties. In addition, the shim-in set-up produced lower TS but higher CT peak load than the no-shim one. The average values of both measured peak load and energy absorption are shown in Figure 11 for comparison. In the TS tests, the shim-out, no shim and shim-in set-ups corresponded to the TS peak loads of 23,16 , and $12 \mathrm{kN}$ and the values of energy absorption as 39,14 , and $11 \mathrm{~J}$, i.e., the shim-out set-up produced the best TS mechanical properties but the shim-in one the worst. In the CT tests, the shim-out, no shim and shim-in setup corresponded to the peak loads of 7,2 , and $3 \mathrm{kN}$ and the

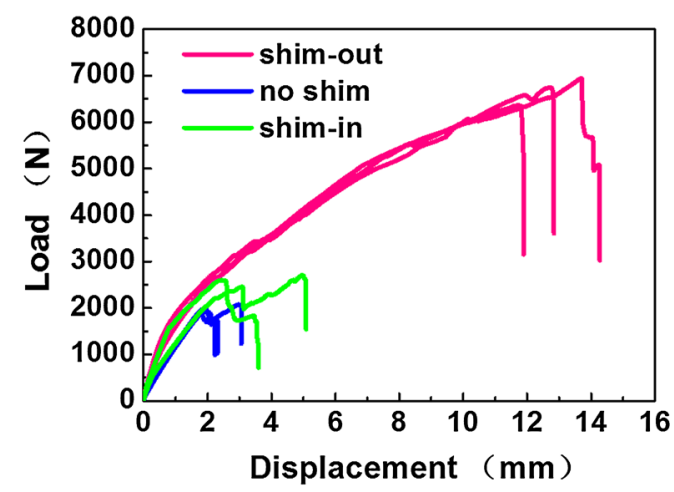

(b)

Fig. 10-The load-displacement curves of spot welded 7Mn steel during the tensile-shear tests $(a)$ and the cross-tension tests $(b)$. 


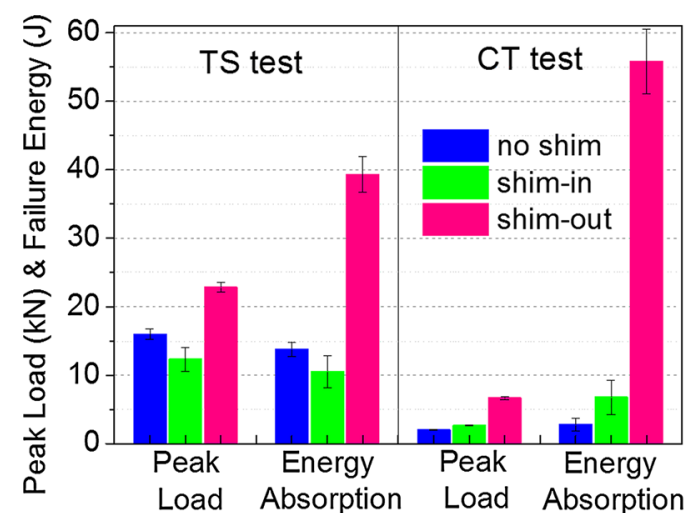

(a)

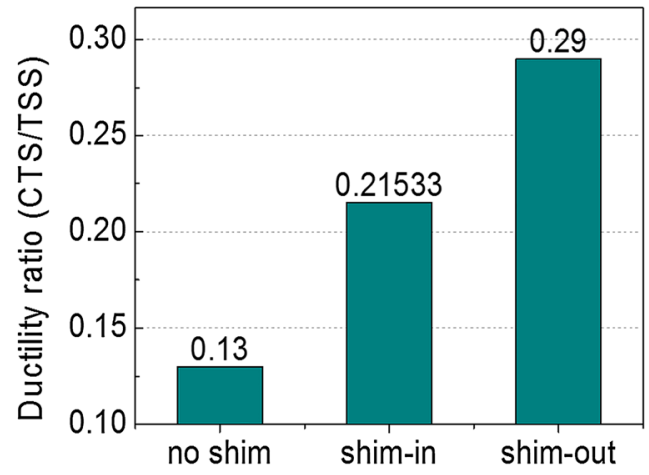

(b)

Fig. 11-The comparison of measured mechanical properties among the three welding set-ups. (a) The average values of peak load and energy absorption, (b) the average values of ductility ratio.
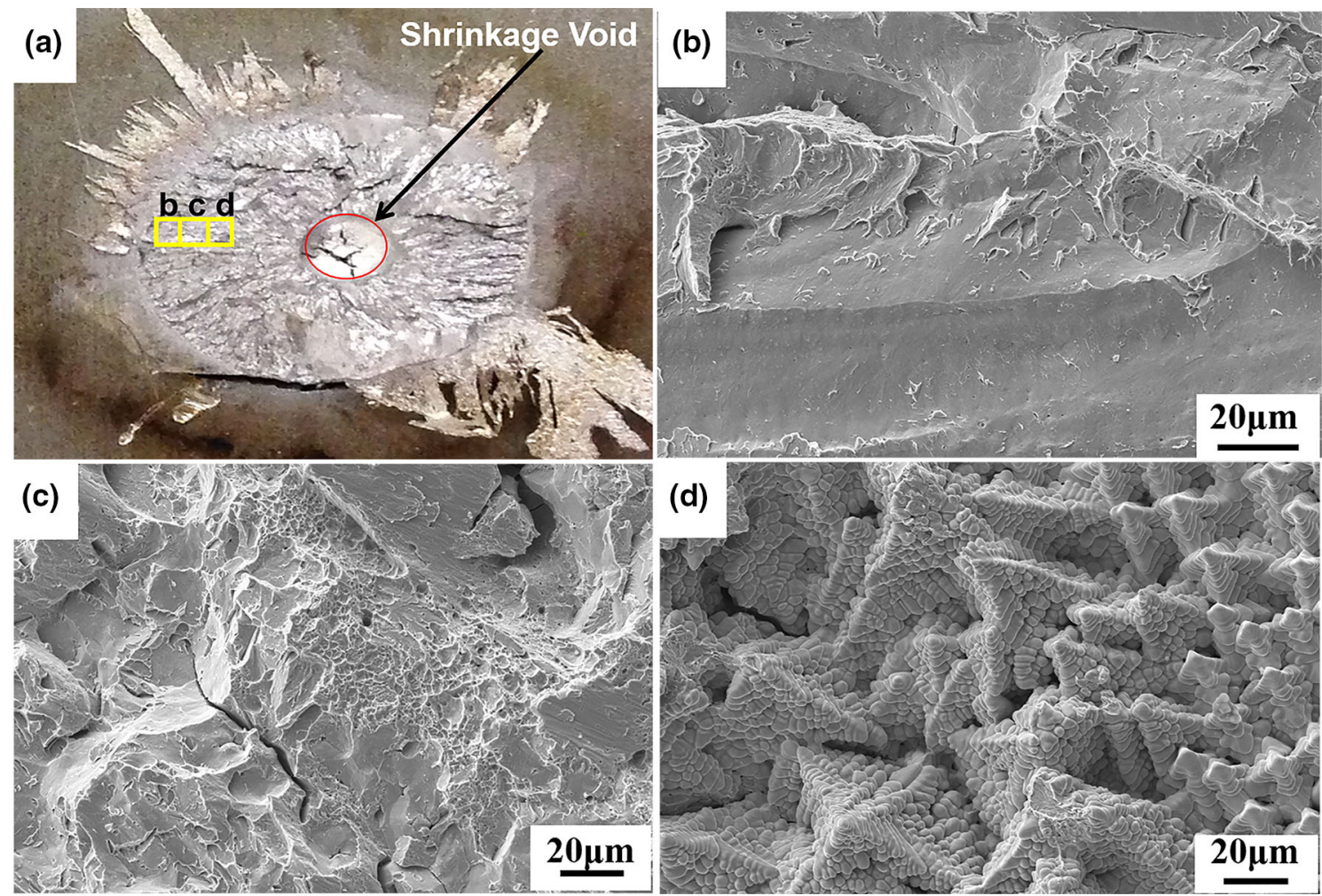

Fig. 12-Interfacial fracture (IF) mode with the features of brittle, ductile and dendrite characteristics in the specimen subjected to the no shim welding. $(a)$ Overall view of the IF, where $(b)$ to $(d)$ locations are magnified and shown, $(b)$ intergranular brittle fracture, $(c)$ transgranular brittle and dimple-like ductile fracture, $(d)$ dendritic fracture.

values of energy absorption as 56,3 , and $7 \mathrm{~J}$, respectively; in this case, the shim-out welding set-up again produced the best mechanical properties but the no shim one was the worst. Moreover, the ductility ratio, which is defined as the ratio of the CT peak load to TS peak load and an indication of the joint ductility, ${ }^{[11]}$ is shown in Figure 11(b). The comparison of ductility ratios among the three set-ups not only confirmed that the shim-out set-up indeed produced the best joint, but also suggested that the shim-in set-up should lead to the better joint ductility than the no shim one, which is consistent with the results of CT tests. The latter shall be attributed to lowest hardness in the FZ of shim-in set-up (Figure 9), resulting from the lowest $\mathrm{C}$ and $\mathrm{Mn}$ contents due to the merging of entire IF steel shim into the $7 \mathrm{Mn}$ steel. 
Table II. The Nugget Size, Fracture Mode and Mechanical Properties of Welds for the Three Welding Set-Ups

\begin{tabular}{llll}
\hline Set-Ups & No Shim & Shim-In & Shim-Out \\
Nugget diameter/thickness (mm) & $7.69 / 1.67$ & $7.67 / 2.38$ & $8.64 / 3.28$ \\
TS test & & & \\
Fracture mode & IF & PF & PIF \& PF \\
Peak load (kN)/extension (mm) & $16.0 / 1.5$ & $12.3 / 1.3$ & $22.8 / 2.7$ \\
CT test & IF & PF & PF \\
Fracture mode & $2.0 / 2.3$ & $2.7 / 3.7$ & $6.7 / 12.7$ \\
Peak load (kN)/extension (mm) & \\
\hline$I F$, interfacial fracture; $P F$, pull-out fracture; $P I F$, partial interfacial fracture; $T S$, tensile-shear; $C T$, cross-tension. \\
\hline
\end{tabular}
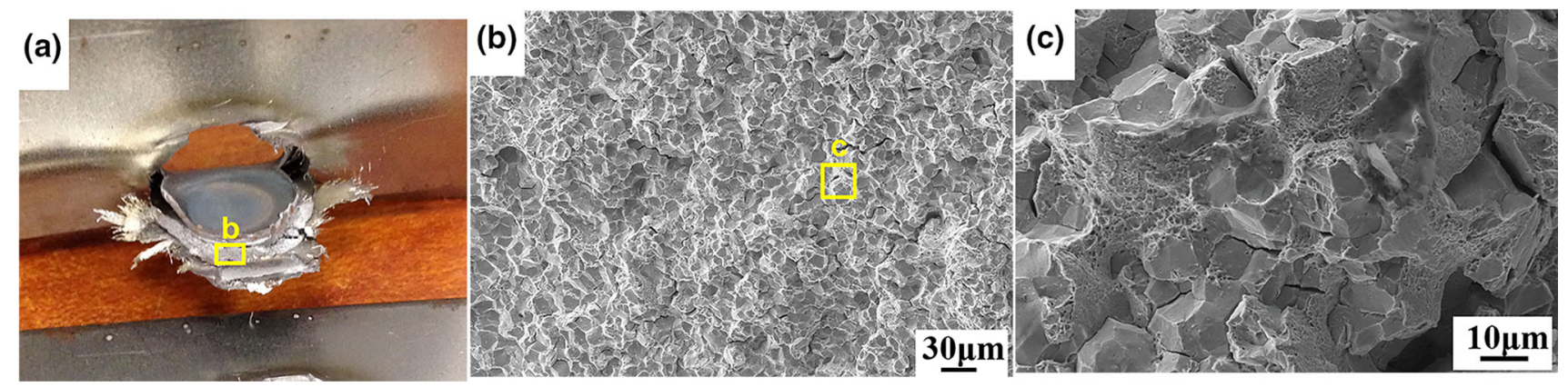

Fig. 13-Pullout fracture (PF) having both the brittle and ductile fracture characteristics in the specimen subjected to the shim-out welding. (a) Overall view of the PF, where ' $b$ ' location is magnified and shown in $(b)$, and further magnified in $(c)$, to show the mixture of both the intergranular brittle and transgranular ductile fracture.

Table III. Thermophysical Properties of $7 \mathrm{Mn}$ and Interstitial-Free Steel ${ }^{[20-22]}$

\begin{tabular}{lcccc}
\hline Steels & $\begin{array}{c}\text { Electrical Resistivity } \\
\left(10^{-8} \Omega \mathrm{m}\right)\end{array}$ & $\begin{array}{c}\text { Thermal Conductivity } \\
(\mathrm{W} /(\mathrm{m} \mathrm{K}))\end{array}$ & $\begin{array}{c}\text { Coefficient of Thermal Expan- } \\
\text { sion }\left(10^{-6} /{ }^{\circ} \mathrm{C}\right)\end{array}$ & $\begin{array}{c}\text { Solidus Tempera- } \\
\text { ture }\left({ }^{\circ} \mathrm{C}\right)\end{array}$ \\
\hline $7 \mathrm{Mn}$ & $34.5^{*}$ & $22.2^{[21]}$ & $15.6^{*}$ & $\begin{array}{c}\text { Specific Heat }(\mathrm{J} / \\
(\mathrm{kg} \mathrm{K}))\end{array}$ \\
Shim & $10.2^{[20]}$ & $74.7^{[20]}$ & $12.1^{[20]}$ & $1456^{* *}$ \\
\hline
\end{tabular}

*Data measured from our own experiments, **data calculated by the commercial Thermo-Calc software.

\section{Fracture Modes}

Figures 12 and 13 are the typical fracture morphologies after the TS tests. In the case of no shim set-up, the interfacial fracture (IF) was observed, which actually consisted of both the intergranular and transgranular brittle fracture, the dimple-like ductile fracture, the dendritic fracture and the voids due to shrinkage (Figure 13), the latter had a considerable influence on the failure strength of spot welds. ${ }^{[17,18]}$ The fracture modes and mechanical properties of welds in all the three set-ups are summarized in Table II together with nugget sizes. In the case of shim-out set-up, we observed the pull-out fracture $(\mathrm{PF})$, which is the mixture of both the intergranular brittle and a considerable fraction of transgranular ductile fracture. Since the latter can consume much more deformation energy than the brittle fracture, ${ }^{[19]}$ the PF mode exhibits better mechanical properties in general.

\section{DISCUSSION}

In the three welding set-ups, there were actually different kinds and amounts of steel sheets stacked between the electrodes: just two 7Mn steel sheets in the no shim set-up, two $7 \mathrm{Mn}$ steel sheets plus one IF steel sheet in the shim-in one and two $7 \mathrm{Mn}$ steel sheets plus two IF steel shims in the shim-out one, see Figure 2. Since they produced the different nugget sizes and tremendously different weld surface (expulsion, cracking etc. in Figure 5), these should be related to the different thermophysical properties of all the materials between the electrodes in the three set-ups, ${ }^{[1]}$ as also given in Figure 2. Table III summarizes the electrical resistivity, thermal conductivity, thermal expansion coefficients, solidus temperatures and specific heat of both the $7 \mathrm{Mn}$ and IF steels. Compared to the 7Mn steel, the IF steel shim has lower electrical resistivity and higher heat conductivity, which means that less heat shall be 
generated in it and heat could dissipate faster from it to the water-cooled electrode. Moreover, the specific heat of both the IF and 7Mn steels is similar, which means that the same heat input to them should lead to the similar temperature rise.

In the shim-out set-up (Figure 2(c)), the temperature on the weld surface should be lowest among the three set-ups because the heat could dissipate faster from IF steel shim to the water-cooled electrodes due to its higher thermal conductivity and less heat is generated in it due to its lower electricity; moreover, the solidus temperature of IF steel is about $73{ }^{\circ} \mathrm{C}$ higher than that of $7 \mathrm{Mn}$ steel. All these factors lead to the temperature on the weld surface in the shim-out set-up being far below the solidus temperature, i.e., the solid-state IF steel shims with enough strength may exist during welding, which prevented the liquid expulsion. In addition, the IF steel shims outside $7 \mathrm{Mn}$ steel sheets were more ductile because they were still ferrite after the rapid cooling and can accommodate more strain induced by the thermal stress; moreover, they acted as extra thermal barrier, leading to lower cooling rate in $7 \mathrm{Mn}$ steel sheets and then reduced thermal stress. Both may contribute to no cracking observed in the shim-out set-up. In contrast, the $7 \mathrm{Mn}$ steel on the weld surface in both no shim and shim-in set-ups just transformed to the briite martensite during the rapid cooling; in these cases, the stress on the martensite directly imposed by electrodes together with thermal stress may easily cause cracking on the weld surface, as observed in Figure 5.

Among the three set-ups, the shim-out set-up led to the largest nugget size because the heat, including both $Q_{1}$ and $Q_{2}$ in Figure 2(c), was largest and the stacked sheets between the electrodes were thickest. Heat was actually generated from two IF steel shims, two $7 \mathrm{Mn}$ steel sheets and particularly the three interfaces as the relatively high electrical resistance, including two IF/ $7 \mathrm{Mn}$ steel interfaces and one $7 \mathrm{Mn} / 7 \mathrm{Mn}$ steel interface. In contrast, the heat generated in the shim-in set-up was from two $7 \mathrm{Mn}$ steel sheets, one IF steel shim and two IF/7Mn steel interfaces; the heat generated in the no-shim set-up was just from two 7Mn steel sheets and one $7 \mathrm{Mn} / 7 \mathrm{Mn}$ interface, which was smallest among them. In addition, the total thickness of the stacked steel sheets between the electrodes is smallest in the no-shim set-up and largest in the shim-out set-up, it is logical that the thickness of nugget formed during welding is smallest in the former case and largest in the latter, as shown in Figure 6.

The mechanical performance of resistance spot welds may depend on many factors, such as nugget size, microstructure, base metal strength etc. ${ }^{[23]}$ However, the nugget size is the most important parameter governing the mechanical properties of spot welds. ${ }^{[13]}$ In our case, the TS peak loads and extensions appeared to be determined mainly by the nugget diameters, whilst the CT peak loads and extensions were determined by the nugget thickness (Table II). This is logical since the peak load shall depend on how much volume of nugget can bear the load along the loading direction. This is also consistent with previous research results. For examples, some models for estimating the TS and CT strength of weld indicate that the peak load shall be in the square proportional to the nugget diameter in the interfacial fracture mode and in the direct proportional to nugget diameter in the pullout fracture mode. ${ }^{[24-26]}$

It is worthy of mentioning that the shim-assisted welding technology has been investigated before. For an example, Peterson ${ }^{[27]}$ inserted a low carbon steel shim at the faying interface of $1330 \mathrm{MPa}$ martensitic steel sheets, i.e., the shim-in set-up in this study, which produced a pull-out fraction and dramatically improved the peak load by over 300 pct. However, the same welding set-up did make any improvement in our case. This is probably because the studied 7Mn steel contains a much higher Mn content than the ordinary martensitic steel; thus, the dilution of $\mathrm{C}$ and $\mathrm{Mn}$ in the fusion zone by IF steel shim is not enough to bring down the $\mathrm{C}$ and $\mathrm{Mn}$ contents for avoiding martensitic transformation. This can be hinted by the observed martensite microstructure in the FZ of shim-in set-up, as shown in Figure 8.

Instead, we invented a new welding setup, i.e., shim-out set-up, which has been not presented before, to our best knowledge. Compared to the no-shim set-up, this novel welding set-up led to the increase of TS peak load by 50 pct (from 16 to $23 \mathrm{kN}$ ) and the increase of CT peak load by three 3 times (from 2 to $7 \mathrm{kN}$ ). Such a dramatical improvement is mainly attributed to the much larger diameter and higher thickness of nuggets achieved in this new set-up, which leads to the pull-out fracture.

\section{CONCLUSIONS}

In this paper, three resistance spot welding set-ups, including no shim, an IF steel shim at the faying interface (shim-in) and shims against the electrodes (shim-out), were employed to investigate the weldability of Fe-7Mn-0.14C medium-Mn steel. The effect of IF steel shims at different locations on the mechanical performance of weld was evaluated by the tensile-shear, cross-tension and microhardness tests, and analyzed through the morphologies and microstructures of welds. The following conclusions can be drawn:

1. Both no shim and shim-in welding set-ups produced the expulsion and cracking on the weld surface, whilst the shim-out set-up led to no expulsion and good surface quality because the IF steel shim can maintain its solid state with a certain strength during welding due to its higher solidus temperature and better thermal conductivity than the medium Mn steel.

2. Compared to the no-shim set-up, our invented shim-out welding set-up has significantly improved the overall mechanical properties of RSW of medium-Mn steel, including peak load, extension, energy absorption and ductility ratio. In particular, this led to the increase of TS peak load by 50 pct and the increase of CT peak load by three times. Such a dramatical improvement is mainly attributed to much larger nugget achieved in this set-up, which leads to the pull-out fracture.

3. Compared to the no-shim set-up, the shim-in welding set-up did not improve the tensile-shear peak load 
and the energy absorption of the medium-Mn steel welds, which is out of our expectation. This is likely because the studied medium-Mn steel contains much higher Mn content than the conventional low-alloyed martensitic steel so that the microstructure in the FZ is still the brittle martensitic phase.

4. The failure mode of the welds transformed from the interfacial fracture in the no-shim welding set-up to the desired nugget-pull-out fracture in the shim-out set-up due to the much larger nugget formed in the later case. This was because more heat was generated due to higher resistivity and more material was involved to melt during welding in the shim-out set-up to feed the larger nugget.

\section{ACKNOWLEDGMENTS}

The authors gratefully acknowledge the financial support and technical support provided by Hundred-Talent program of Chinese Academy of Sciences and General Motors.

\section{OPEN ACCESS}

This article is distributed under the terms of the Creative Commons Attribution 4.0 International License (http://creativecommons.org/licenses/by/4.0/), which permits unrestricted use, distribution, and reproduction in any medium, provided you give appropriate credit to the original author(s) and the source, provide a link to the Creative Commons license, and indicate if changes were made.

\section{REFERENCES}

1. H. Aydin, E. Essadiqi, I.H. Jung, and S. Yue: Mater. Sci. Eng. $A, 2013$, vol. 564, pp. 501-08.
2. J.T. Benzing, J. Bentley, J.R. McBride, D. Ponge, J. Han, D. Raabe, and J.E. Wittig: Microsc. Microanal., 2017, vol. 23 (S1), pp. 402-03.

3. B. Hu, H.W. Luo, F. Yang, and H. Dong: J. Mater. Sci. Technol., 2017, vol. 33 (12), pp. 1457-64.

4. Z.H. Cai, H. Ding, R.D.K. Misra, and Z.Y. Ying: Acta Mater., 2015, vol. 84, pp. 229-36.

5. H.W. Luo, J. Shi, C. Wang, W. Cao, X. Sun, and H. Dong: Acta Mater., 2011, vol. 59 (10), pp. 4002-14.

6. M.J. Merwin: Proc. SAE Intl. World Congr. Exhib., 2007, vol. 1, p. 336.

7. J. Yoo, K. Han, Y. Park, and C. Lee: Mater. Chem. Phys., 2014, vol. 146 (1-2), pp. 175-82.

8. X.J. Di, S.J. Deng, and B.S. Wang: Mater. Des., 2015, vol. 66 (A), pp. 169-75.

9. N. Lun, D.C. Saha, A. Macwan, H. Pan, L. Wang, F. Goodwin, and Y. Zhou: Mater. Des., 2017, vol. 131, pp. 450-59.

10. X.J. Di, M. Li, Z.W. Yang, B.S. Wang, and X.J. Guo: Mater. Des., 2016, vol. 96, pp. 232-40.

11. R. Ashiri, S.P.H. Marashi, and Y.D. Park: Weld. J., 2018, vol. 97, pp. 157-69.

12. S. Agashe and H. Zhang: Weld. J., 2003, vol. 82 (7), pp. 179-83.

13. M. Pouranvari and S.P.H. Marashi: Sci. Technol. Weld. Join., 2013, vol. 18 (5), pp. 361-403.

14. W. Li: PhD thesis, University of Michigan, USA, 1999.

15. D.C. Saha, S. Han, K.G. Chin, I. Choi, and Y.D. Park: Steel Res. Int., 2012, vol. 83 (4), pp. 352-57.

16. W. Peterson: Sheet Metal Welding Conference X, 2006.

17. H. Zhang and J. Senkara: Resistance Welding: Fundamentals and Applications, CRC Press, Boca Raton, 2011.

18. X. Deng, W. Chen, and G. Shi: Finite Elem. Anal. Des., 2000, vol. 35 (1), pp. 17-39.

19. J. Mendez, M. Ghoreshy, W.B.F. Mackay, T.J.N. Smith, and R.W. Smith: J. Mater. Process. Technol., 2004, vol. 153, pp. 596602 .

20. Z. Wan, H.P. Wang, M. Wang, B.E. Carlson, and D.R. Sigler: Int. J. Heat Mass Transf., 2016, vol. 101, pp. 749-63.

21. J.K. Guo, J.L. Song, J. Fu, J.X. Cao, J.M. Hou, and R.H. Sun: Spec. Cast. Nonferrous Alloys, 2014, vol. 34 (10), pp. 1050-53.

22. Q.Z. Shen and J.M. Du: Fundamentals of metallurgical transpot, Press of Metallurgical Industry, Beijing, 2009.

23. L. Deng, Y. Li, B.E. Carlson, and D.R. Sigler: Weld. J., 2018, vol. 97 (4), pp. 120-32.

24. Y.S. Yang, K.J. Son, S.K. Cho, S.G. Hong, S.K. Kim, and K.H. Mo: Sci. Technol. Weld. Join., 2001, vol. 6 (6), pp. 397-401.

25. H. Oikawa, G. Murayama, T. Sakiyama, Y. Takahashi, and T. Ishikawa: Shinnittetsu Giho, 2006, vol. 39, pp. 39-45.

26. Y. Sakuma and H. Oikawa: Shinnittetsu Giho, 2003, vol. 384, pp. 30-38.

27. W. Peterson: ICAWT 1997 International Conference, Advances in Welding Technology, Columbus, 1997, pp. 17-19. 